\title{
LA BIOLOGIE DE LAMARCK
}

\author{
Goulven Laurent \\ Université Catholique del' Ouest - Bibliothèque Centrale - 3, Place André-Leroy-49008 Angers
}

\section{Invention du mot Biologie. Une nouvelle science}

Il est significatif que ce soit dans les toutes premières années qui ont suivi son invention de la théorie du Transformisme (ou de l'Evolution), que Lamarck ait éprouvé le besoin de créer un mot nouveau pour désigner l'étude de la vie. Le rapprochement des dates de ces deux événements dans l'oeuvre de Lamarck est en effet assez suggestif: 1800: première proclamation et première formulation de la théorie de la transformation des espèces; 1801 (1802 cf note2): première proposition du mot "Biologie".

Lamarck avait conscience de professer une nouvelle représentation du monde animé quand il défendait sa "conclusion particulière"1, opposée à la "conclu-sion admise jusqu'à ce jour", sa vision transformiste opposée à la vision fixiste et créationniste de Cuvier.

Cette vision révolutionnaire de la transformation historique des êtres vivants allait entraîner immédiatement un autre élargissement de la pensée du naturaliste. II étend sa "physique terrestre" à l'origine des êtres vivants, et, par conséquence, à celui de l'apparition de la vie elle-même sur la terre. En raccordant les êtres entre eux dans une vision de passages graduels des uns aux autres, Lamarck entrevoit en effet la possibilité de les relier, à leur base, directement avec la matière ellemême.

Du coup la vie se relie à la physique, et si la physique est déjà depuis longtemps le champ d'une science spécifique et bien constituée, la vie elle-même acquiert aussi le mëme statut. Cette science n'existait pas encore, car elle vient de naître: aussi le mot pour la désigner n'existait pas davantage. En participant à la création de la science de la vie, Lamarck éprouve tout naturellement le besoin de ecréer le mot pour désigner cette nouvelle discipline: il propose donc le mot Biologie.

C'est en janvier 1802 (ou decembre $1801^{2}$, mais, de toute manière, comme le livre paralt en janvier 1802, la date de 1801 peut être retenue), dans l'Hydrogéologie ${ }^{3}$, que

1 Lamarck, J.B. (1809), Philosophie zoologique, Dentu, Paris, 2 vol., I, pp.265-6.

2 Cf. MÜLlER G.H. (1983), "First use of biology", Nature, 1983, vol.302, p.744.

3 LAMARCK, J.B. (1802), Hydrogéologie, chez Agasse et Maillard, Paris, p.7. 
GOULVEN LAURENT

Lamarck emploie ce mot pour la première fois, dans un cadre de disciplines scientifiques embrassant l'ensemble des phénomènes naturels: "Une bonne Physique terrestre doit comprendre toutes les considérations du premier ordre, relatives à l'atmosphère terrestre; ensuite toutes celles du même genre, qui concernent l'état de la croute externe de ce globe, ainsi que les modifications et les changemens qu'elle subit continuellement; enfin celles de la même sorte, qui appartiennent à l'origine et aux développemens d'organisation des corps vivans. Ainsi toutes ces considérations partagent naturellement la physique terrestre en trois parties essentielles, dont la première doit comprendre la théorie de l'atmosphère, la Météorologie; la seconde, celle de la croute externe du globe, l'Hydrogéologie; la troisième enfin, celle des corps vivans, la Biologie". II est à signaler que, dans cette première mention de ce mot, Lamarck ne signale pas qu'il est nouveau ${ }^{4}$. Quelques mois plus tard, dans ses Recherches sur l'organisaition des Corps vivans ${ }^{5}$, Lamarck se contente encore de signaler que c'est le nom qu'il envisage de donner à une étude qu'il a l'intention d'entreprendre, et dont il donne la définition ${ }^{6}:$ "elle comprend tout ce qui a rapport aux corps vivans, et particulièrement à leur organisation, à ses développemens, à sa composition croissante avec l'exercice prolongé des mouvemens de la vie, à sa tendance à créer des organes spéciaux, à les isoler, à en centraliser l'action dans un foyer, etc." Un exposé structuré et détaillé de cette nouvelle discipline est resté un de ses objectifs, mais il n'a pas eu le temps de le réaliser. Lamarck en reparle en effet en 1809, en introduisant sa Philosophie zoologique "cette Philosophie zoologique présente les résultats de mes études sur les animaux, leurs caractères généraux et particuliers, leur organisation, les causes de ses développemens et de sa diversité, et les facultés qu'ils en obtiennent; et pour la composer, j'ai fait usage des principaux matériaux que je rassemblois pour un ouvrage projeté sur les corps vivans, sous le titre de Bioiogie; ouvrage qui, de ma part, restera sans exécution".

En $1815^{8}$, Lamarck n'a pas encore totalement abandonné son projet: "les corps vivans", écrit-il, "offrent en eux, et dans les phénomènes divers qu'ils présentent, les matériaux d'une science particulière qui n'est pas encore fondée, qui n'a pas mêrne de nom, dont j'ai proposé quelques bases dans ma Philosophie zoologique, et à laquelle je donnerai le nom de Biologie". II considère encore en 1816 que "la blologien'est

\footnotetext{
4 Cf. note 2.

5 LAMARCK, J.B. (1802), Recherches sur l'organisation des Corps vivans, chez Maillard, Paris, p. 186.

6 Ibid., p.203.

7 LAMarck, J.B. (1809), Philosophie zoologique, i, p.XViii.

8 LAMARCK, J.B. (1815-1822), Histoire Naturelle des Animaux sans Vertèbres, Verdière, Paris, 7 vol., I, Introduction, p.49.
} 
pas réellement fondée, et qu'on n'a même pas pensé à en faire un sujet d'étude particulière"9.

\section{La conception préscientifique}

La date de 1801(1802) où nous avons rencontrée pour la première fois le mot Biologie chew Lamarck nous montre que son auteur n'avait pas eu l'idée d'une escience nouvelle avant cette date -il avait déjà près de 60 ans. Cependant il avait déjà traité de questions concernant les êtres vivants, mais dans une perspective non ou pré-scientifique.

Lamarck s'était affirmé matérialiste dès le début de ses réflexions sur la nature. Cependant, dans les prernières formulations de son système, il n'avait pas réussi à y intégrer les vivants. II restait donc une sorte de lacune ou d'inconséquence dans sa construction intellectuelle. Son cas n'était d'ailleurs pas isolé: ce sera celui de bon nombre de naturalistes tout au long du xixe siècle, et il le reste encore aujourd'hui.

En 1794, dans un de ses premiers écrits, Lamarck place en effet une différence fondamentale entre le vivant et le non vivant. Dans ses Recherches sur les Causes des principaux faits physiques, dont on sait que la première rédaction remonte en fait à $1776^{10}$, Lamarck souligne le fossé qui les sépare ${ }^{11}$ : "Tous les êtres qui font partie du globe que nous habitons, sont évidemment distingués en deux classes générales, et diffèrent tellement entre eux à cet égard, qu'ils ne sont nullement comparables: ce sont les êtres doués de la vie, et tous ceux qui en sont dépourvus". Un peu plus loin dans le même ouvrage, Lamarck dénonce l'attitude de certains naturalistes ${ }^{12}$ "qui ont regardé comme possible la formation d'une chaîne non interrompue dans laquelle seroient compris tous les êtres qui sont dans la nature, et qui par conséquent ont pensé qu'on pouvoit, par un passage insensible, lier les minéraux aux êtres organiques, comme s'il y avoit quelque rapport entre un être doué de la vie... et... un morceau de minéral".

A cette époque, Lamarck considère donc qu'il y a un fossé infranchissable entre la vie et la matière, entre le vivant et le non vivant. II soutient en effet l'existence d'un "principe vital"13, qui est "la cause active qui a la faculté de modifier la matière et de

\footnotetext{
9 «Des rapports qui doivent être employés dans la distribution et la classification des animaux», (1816-1817), dans VACHON, M., Rousseau, G. et Laissus, Y. (eds)(1972), J.-B. Lamarck Inédits, Paris, p.284.

10 LAMARCK, J.B.(1794), Recherches sur les Causes des principaux faits physiques, Paris, 2 vols, i, p.vii, ii, p.378.

11 Ibid., ii, p.285.

12 Ibid., p.378.

13 Ibid., p.30.
} 
combiner immédiatement les élémens". Et, assure-t-il14 : "nous croyons qu'il n'est pas possible qu'une cause physique quelle qu'elle soit, ait jamais pu donner lieu à l'existence des êtres organiques; et en un mot, nous pensons que les diverses sortes de matières qui existent, n'ont pu dans telles circonstances qu'on pourroit imaginer, produire un seul composé vraiment doué de la vie. Ainsi, ce qui constitue l'essence de la vie d'un être organique, est vraisemblablement un principe à jamais inconcevable à l'homme, ou au moins un principe dont la connoissance paroît devoir aussi bien échapper à ses recherches physiques, que celle de la cause de l'existence de la matière et l'activité générale répandue dans la nature". Les êtres vivants échappent à la matière par leur nature même de vivants: ils "jouissent d'un principe particulier, dont sans doute l'origine et l'essence ne peuvent être assignées physiquement"15.

Jusqu'en 1797 -alors qu'il est en train de mettre en place un tableau du règne animal $^{16}$ qui va le faire déboucher dans le transformisme, alors qu'il affirme ${ }^{17}$ "qu'il existe dans l'organisation de ces différens êtres vivans, une graduation remarquable depuis la simplification d'organisation la plus grande, jusqu'à I'organisation la plus compliquée à tous égards"- Lamarck soutient encore une vision dualiste et antagoniste de la vie et de la matière. La position qu'il tient dans ses Mémoires de Physique et d'Histoire naturelle est en effet sans équivoque $^{18}$ : "II n'y a certainement aucune union, aucune nuance à découvrir entre les êtres vivans et les corps bruts et inorganiques", et il renvoie expressément à ses affirmations de 1794. "C'est donc sans fondement qu'on a dit qu'il n'y a point de sauts dans la nature". "Tous les êtres qui font partie du globe que nous habitons, répète-t-il, sont évidemment partagés en deux règnes distincts; le règne des êtres vivans, celui qui comprend tous les êtres doués de la vie, et le règne des corps bruts, celui auquel se rapportent tous les corps et toutes les matières qui sont dépourvus de la vie"19.

Et il continue: "On peut dire qu'il y a en quelque sorte une distance infinie entre lès êtres qui composent ces deux règnes, et qu'ils diffèrent tellement entr'eux, qu'ils ne sont pas même comparables, c'est-à-dire qu'ils ne doivent pas etre présentés comparativement sur une même ligne en forme de chaîne continue, comme on l'a cru long-temps, parce que la continuité de cette chaîne est une erreur évidente".

A ce moment de sa réflexion, Lamarok ne peut encore évidemment attribuer l'origine des êtres vivants qu'à une puissance extérieure à la nature ${ }^{20}$ : "lorsque j'ai parlé

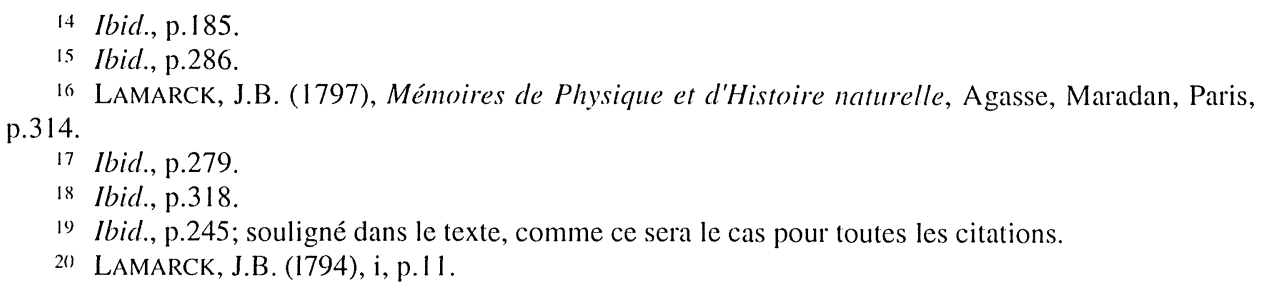


des êtres organiques, assure-t-il, et que j'ai dit que c'étoit à eux seuls qu'étoient dus tous les composés qui existent dans notre globe, je ne me suis pas permis de remonter à l'origine du monde pour rendre raison comment les premiers de ces ëtres vivans, ont pu exister eux-mêmes; et pour savoir si à cette époque, il se trouvoit, ou non, des minéraux. Car je ne suis pas plus en état d'expliquer physiquement ce qui concerne l'époque dont il s'agit, que de déterminer l'état de la nature dans ces tems inconnus. C'est pourquoi, sans m'arrêter à ce qui a pu être, et qui n'est pas en mon pouvoir de vérifier convenablement, je me suis borné dans mes recherches à l'observation de la nature, telle qu'elle est actuellement, et à l'examen des phénomènes nombreux et infiniment variés, qu'elle nous présente de toutes parts". Pour Lamarck, les problèmes d'origine sont des problèmes qu'il faut laisser de coté, car il y a des "connoissances ... que l'homme raisonnant philosophiquement, ne me paroït jamais pouvoir acquérir"21. Parmi ces connaissances, Lamarck cite expressément "celle de l'existence des êtres organiques et de ce qui constitue la vie et l'essence de ces êtres; car la matière, avec toutes ses qualités, ne me paroît nullement capable de produire un seul être de cette natures". Lamarck soutient encore ${ }^{22}$ que "l'essence d'un être vivant n'est pas constituée par le nombre des organes, ni par la possession unique de tel ou de tel organe particulier, mais vraiment par la jouissance d'un principe ou mouvement vital qui ne nécessite que les organes essentiels à son existence". II importe de souligner la clarté et la netteté des affirmations de Lamarok à ce stade préscientifique de ses réflexions sur les vivants ${ }^{23}$ : "j'ai osé avancer en débutant, affirme-t-il, que l'existence de ces êtres étonnans" (les "êtres organiques"), "n'appartenoit nullement à la nature; que tout ce qu'on peut entendre par le mot nature, ne pouvoit point donner la vie, c'est-à-dire, que toutes les facultés de la matière, jointes à toutes les circonstances possibles, et mëme à l'activité répandue dans l'univers, ne pouvoient point produire un être muni du mouvement organique".

II est intéressant et important de noter cependant la distinction que fait déjà Lamarck entre l'origine et le fonctionnement du vivant. On ne peut s'empêcher de penser ici à la distinction que faisait Newton entre le lancement et le fonctionnement du mouvement de l'univers. Newton ne prétendait en effet qu'à expliquer son fonctionnement. Et il le faisait en faisant appel uniquement à des lois physiques c'est le sens de sa fameuse formule: "hypotheses non fingo".

II en est de même en quelque sorte en ce qui concerne Lamarck. Une fois que la vie est lancée - ou plus exactement que le vivant est doté de la vie- le vivant est à même de fonctionner selon des lois physiques. On sent que s'il y a des lacunes dans

21 Ibid., ii, p.26, note 1.

22 Ibid., p. 190.

2.3 Ibid., p.213. 
son système, le jour où il sera à même de les combler, Lamarck le fera aussi selon le mëme principe de l'application de lois physiques naturelles.

En effet, à y regarder de plus près, l'affirmation d'un principe totalement étranger à la nature n'a jamais été isolée chez Lamarck des conditions de son fonctionnement. Même dans cet état d'esprit, Lamarck — cohérence intellectuelle oblige — soutient en 1794 I'existence d'une union étroite, dans le vivant, entre la vie et la matière ${ }^{24}$ : "s'il est vrai, comme nous venons de le dire, que le principe inconcevable qui fait l'essence de la vie, soit assez peu dépendant de la nature, pour que la matière aidée du concours de toutes les circonstances possibles, n'ait jamais pu le produire; il n'en est pas moins vrai en même tems que l'étonnant principe dont il s'agit, ne peut absolument pas exister physiquement sans la matière".

En 1797 Lamarck parle moins d'un "principe vital" extérieur à la nature que d'un "mouvement vital" dépendant de l"organisation -ce concept dont on sait qu'il est en train de devenir fondamental dans l'étude et la mise en ordre du règne animal qu'il est en train de réaliser. II souligne maintenant plus fortement ${ }^{25}$ que "la vie ou le mouvement organique ne peut exister que par les relations actives des solides avec les fluides", c'est-à-dire des relations entre les différents éléments du corps vivant ${ }^{26}$.

Après avoir bien séparé les corps vivants des corps non vivants, Lamarck définit les premiers en mettant l'accent beaucoup plus sur le mouvement en luimême que sur le principe du mouvement. Ils jouissent "d'un mouvement particulier que je nomme mouvement organique; mouvement dont la possibilité constitue ce qu'on nomme la $v i e^{127}$. Lamarck caractérise l'être vivant comme "un corps naturel, organisé, composé de diverses sortes de parties qui agissent et réagissent les unes sur les autres, sont tenues plus ou moins en mouvement, soit par les suites même de leur action réciproque, soit par une cause extérieure; et qui subissent des changemens continuels dans leur nature, par l'effet immédiat de leur mouvement et de leur action"28.

La "cause extérieure" à laquelle Lamarck fait allusion dans le cas présent est, non pas le "principe vital", insufflé aussi bien sûr de l'extérieur, mais l'action des "fluides", caloriques ou électriques. Et Lamarck, pour bien se faire entendre, met distinctement en équation "la vie" et "tout mouvement organique qui la constitue"29.

24 Ibid., p. 186.

25 LAMARCK, J.B. (1797), p.279.

26 Expression à rapprocher de celle de Cuvier: "Un corps organisé, comme une plante, un animal, est composé d'un tissu de solides qui contiennent des fluides en mouvement. Toutes ses parties ont une action réciproque les unes sur les autres, et concourent à un but commun, qui est l'entretien de la vie", Cuvier, G. (1798), Tableau élémentaire de l'Histoire naturelle des Animaux, Baudoin, Paris, p.5.

27 LAMARCK, J.B. (1797), p.246.

28 Ibid., p.249.

29) Ibid., p.250, note 1 . 
Ainsi, de manière de plus en plus expresse, Lamarck réduit la définition et le rôle de la vie à sa fonction: "je pense donc que la vie, dans les êtres qui en sont doués, n'est autre chose que le mouvement qui résulte, dans les parties de ces êtres, de l'exécution des fonctions de leurs organes essentiels" 30 .

\section{Fonctionnement des êtres vivants}

Le principe vital est nécessaire pour mettre la machine vivante en route. Mais ensuite cette machine trouve dans son propre mouvement et dans les influences extérieures les éléments nécessaires à son fonctionnement et à son perfectionnement. Lamarck a en effet, comme nous l'avons dit, la même vision de la marche de l'être vivant que Newton dans la marche des astres. II est clair que pour l'un et l'autre il faut un choc de départ: I'attraction, assure Lamarck, ne suffit pas à faire marcher l'univers, "il faut encore supposer une impulsion particulière, ou un mouvement de projection communiqué à ces corps" ${ }^{131}$. Ensuite le reste marche tout seul.

II en est de même pour le vivant. Une fois que le "mouvement" est donné par le principe vital, la machine vivante fonctionne mécaniquement et, pour ainsi dire, automatiquement. L'être vivant tel que le conçoit Lamarck est en effet un être composé d'organes, doués de mouvement, c'est-à-dire de facultés. Le "fluide calorique", nécessaire au point de départ pour que le principe vital puisse s'exercer, I'est ensuite continuellement pour entretenir le mouvement de la vie: "lorsqu'un être vivant animé se trouve dans la sphère active de ce fluide en expansion, toutes les parties de son corps, et particulièrement celles qui forment sa surface extérieure, sont bientôt pénétrées par ce fluide qui, en s'étendant, ne surmonte les obstacles que les corps environnans font à sa dilatation, qu'en pénétrant ces mêmes corps, et les modifiant par sa force expansive" 32 .

Une fois la vie lancée, en effet -et c'est sans doute important- Lamarck se fait fort d'expliquer tout le reste par l'action des fluides. Le principal en est le feu pour Lamarck -mais il I'est aussi pour tout le monde, même aujourd'hui- la chaleur, ou, comme il le nomme, le calorique: mais ce n'est qu'une question de vocabulaire, auquel l'historien des sciences doit se faire. II est constamment entretenu sur terre par le solei $^{33}$. La nature ne risque pas d'en manquer: "En effet, cette cause'agit continue-

Ibid., p.255.

31 LAMARCK, J.B. (1794), ii, p.27, note 1.

32 Ibid., i, p.328.

33 Ibid., p.111. 


\section{GOULVEN LAURENT}

llement, quelles que sobent ses modifications qu'opèrent la plus ou moins grande distance de l'astre qui la forme, ou ses divers aspects à cet égard..."34 .

L'action de la chaleur est fondamentale dans ce qu'on peut appeler le fonctionnement de la vie. Lamarck ne fait d'ailleurs pas de distinction entre le feu et l'électricité. Pour lui, ce sont deux aspects d'une même réalité35. L'électricité, assure-t-il, "parô̂t constituer un fluide fort analogue à celui du feu, si toutefois ce n'est pas la même matière, mais observée dans des circonstances qui lui donnent des qualités et des propriétés particulières..." "L'identité de ces matières, ajoute-t-il, semble se confirmer tous les jours par un grand nombre de propriétés qui leur sont communes. Une des plus remarquables, par exemple, est celle qu'a fait connoître Franklin, lorsqu'il prouve que les corps qui conduisent parfaitement bien la matière électrique, sont aussi de fort bons conducteurs de la matière du feu"36. Lamarck va même jusqu'à se montrer disposé à réunir dans un même concept les trois éléments: le feu, l'électricité et le fluide magnétique"37. "Par quelques modifications inconnues", assure-t-il en parlant du feu, "il constitue peut-être $1^{\circ}$ le fluide électrique, $2^{\circ}$ le fluide magnétique".

Le calorique est en effet le moteur efficace de toute vie ${ }^{38}$ : "Il existe dans la nature une cause particulière, puissante et continuellement active, qui a la faculté de former des combinaisons, de les multiplier, d'en diversifier la nature, et qui tend sans cesse à les surcharger de principes et à en augmenter les proportions jusqu'à un certain terme". A ce stade, cette activité peut concerner tous les corps, organiques ou inorganiques.

Mais c'est dans son application à la vie que son rôle est le plus remarquable. II faut bien distinguer les deux moments de l'histoire de l'être vivant: celui de son apparition, et celui de son fonctionnement ${ }^{39}$. En effet, si le principe vital "est vraisemblablement" (noter la réserve implicite) "un principe à jamais inconcevable à l'homme, ou au moins un principe dont la connoissance parô̂t devoir aussi bien échapper à ses recherches physiques, que celle de la cause de l'existence de la matière et l'activité générale répandue dans la nature", la deuxième phase ne repose pas sur les mêmes principes: "II n'en est pas de même, à ce qu'il nous semble, de la cause physique qui entretient la vie des êtres organiques, de celle qui donne lieu à leur développement, et enfin de celle qui produit leur mort inévitable: les facultés de l'homme, son génie, et les connoissances dont il est vraiment susceptible, lui permet-

34 Ibid., p. 257

35 Ibid., p.199, note 1.

36 Ibid., p.202

37 LAMARCK, J.B. (1796), Réfutation de la théorie pneumatique, ou de la nouvelle doctrine des chimistes modernes, Paris, p.36; cf. aussi LAMARCK, J.B. (1797), p.142.

38 LAMARCK, J.B. (1797), p.101.

39) LAMARCK, J.B. (1794), ii, p. 185. 
tent sans doute de porter jusques là ses recherches, et de faire d'autres tentatives pour pénétrer ces secrets importans".

On sent bien que, dès cette étape de sa réflexion, Lamarck était impatient d'aller plus loin sur la voie de la recherche de la nature de la vie. Les développements que nous rencontrerons plus loin ne seront donc pas un horsd'oeuvre dans la pensée de Lamarck, ni dans le perfectionnement de son système. Quand il a fait remarquer que le principe vital ${ }^{40}$ "ne peut absolument pas exister physiquement sans la matière", et qu'il n'a même pas hésité à mettre en équivalence "principe" et "mouvement vital"4l le glissement de mots n'était pas innocent. S'il répète que ${ }^{42}$ la cause qui entretient la vie "n'est point dans l'essence de la matière..., mais (qu') elle réside évidemment dans le principe de la vie!!; il précise "... dans les facultés du mouvement vital, en un mot dans les fonctions organiques": toujours ce glissement d'un principe extérieur à son activité bien inscrite dans les organes physiques.

Lamarck envisage meme déjà que le développement de la vie est dû à l'influence d'agents indépendants du principe vital. II existe en effet "beaucoup de variétés produites par l'effet des circonstances"43.

On peut dire par conséquent que la plus grande partie de la philosophie biologique de Lamarck est déjà en place à cette époque. Lamarck semble à ce moment ne faire qu'une concession pour ainsi dire forcée à l'ignorance et à l'impertection du savoir qui l'obligent à laisser une place à l'action d'un créateur dans l'origine de la vie. Nous savons qu'il ne sera pas le seul à se faire accorder cette concession. Cinquante ans plus tard Darwin le fera à son tour, marquant ainsi un recul notoire par rapport à Lamarck, qui lui sera reproché par les scientifiques de l'époque ${ }^{44}$.

Alors qu'en certains passages Lamarck accordait une place secondaire à la matière, il semble maintenant lui donner le rôle principal: "S'il est vrai, comme nous venons de le dire, que le principe inconcevable qui fait l'essence de la vie, soit assez peu clépendant de la nature, pour que la matière, aidée du concours de toutes les circonstances possibles, n'ait jamais pu le produire; il n'en est pas moins vrai en même tems que l'étonnant principe dont il s'agit, ne peut absolument pas exister physiquement sans la matière" 45 .

Le "principe vital" est en effet tellement uni à la matière que Lamarck refuse le concept d'une âme spirituelle, ou plutôt il affirme que ce concept n'a rien à voir avec

40) Ibid., p. 186.

41 Ibid., p. 188 .

42 Ibid., p.288.

4.3 Ibid., p. 214.

44 Sur la modernité de Lamarck en biologie, cf. le jugement de Roger, J. (1995): Pour une histoire des sciences à part entière, «Lamarck et la bioiogie», p.299.

45 LAMARCK, J.B. (1794), ii, p. 186. 
la science. "Pour moi, sans rien rejeter de ce qui tient à la croyance religieuse, ni de ce qu'il peut être consolant pour l'homme de bien se persuader, je dirai que ce genre de considération est absolument étranger à mon sujet; parce que l'âme immortelle de l'homme, et l'âme périssable des bêtes, etc. ne peuvent m'être connues physiquement"46. L'on voit que Lamarck avait bien conscience de la séparation épistémologique des différents domaines de la réflexion humaine, et qu'il ne fallait pas les confondre.

L'origine du principe vital est peut-être extérieure à la nature, mais son action se définit physiquement dans son domaine ${ }^{47}$ : "En effet, ce principe incompréhensible réside essentiellement dans un mouvement particulier des organes des êtres qui en sont munis".

Lamarck, ayant fait la concession, nécessaire à son sens, d'un agent externe au point de départ de la vie, ramène ensuite constamment ce phénomène dans le domaine des faits physiques ${ }^{48}$ : "La vie, ce principe inconcevable, n'existe que par les fonctions des organes essentiels à sa conservation; et on peut dire qu'il y a une identité si grande entre ce qu'on entend par principe ou mouvenent vital et par fonction organique, que l'existence de l'un suppose toujours celle de l'autre, et que la nullité de l'une constitue essentiellement l'exclusion de l'autre par la même raison".

Ceux qui auraient voulu, à partir de la concession obligée faite par Lamarck de l'action d'un créateur à l'origine de la vie, maintenir la vie dans le domaine de la métaphysique, en sont donc pour leurs frais ${ }^{49}$ : "Pour réussir à faire connoltre la cause de l'entretien de la vie des êtres organiques, j'ai tâché d'abord de prouver que cette même vie résidoit essentiellement dans un mouvement particulier que j'ai nommé mouvement organique... J'ai fait ensuite remarquer que le mouvement organique s'opéroit d'autant plus facilement dans les organes d'un individu, que les fibres de cet être vivant étoient plus souples et plus flexibles". Tout se ramène donc aux organes, à leur qualité physique, et à leur état de bon fonctionnement.

Le lien constant que Lamarck met entre la vie et le mouvement fait qu'il en vient même à définir l'une par l'autre. La vie et le mouvement deviennent ainsi chez Lamarck le même produit naturel de la matière organisée ${ }^{50}$ : I'être vivant, "par l'effet de la vie dont il est doué, jouit intérieurement de mouvemens particuliers qui résultent des fonctions qu'exécutent ses organes; et... au moyen de l'exécution de ces fonctions organiques, cet être a la faculté de composer sa propre substance, c'est-à-dire, de modifier, de préparer, en un mot, d'assimiler sans cesse les matières dont il se nourrit,

46 LAMARCK, J.B. (1797), p.265.

47 LAMARCK, J.B. (1794), ii, p. 186.

48 Ibid., p.191.

4) Ibid., p. 214

5) Ibid., p. 248 
à la substance même dont il est formé; et de réparer les pertes que la nature lui fait à tout moment subir".

Lamarck éprouve ainsi de plus en plus le besoin de définir le vivant par son action, plus que par son "principe vital". "Un corps vivant, assure-t-il, étant composé de diverses sortes de parties, dont certaines d'entre elles sont tenues en mouvement par une cause, soit interne, soit externe, le mouvement dont il s'agit constitue dans cet etre l'activité particulière qui est essentielle à sa conservation, et en laquelle réside sa vie. Ainsi, tout être ou corps naturel qui ne peut conserver son existence que par la jouissance du mouvement organique que je viens de désigner est un être vivant" 51 .

Nous nous sommes étendu sur cet aspect de la pensée de Lamarck, pour bien montrer comment les éléments essentiels de sa pensée biologique étaient déjà en place dès la phase que nous avons appelée pré-scientifique. L'attention portée à ces textes montre combien la querelle faite depuis à la chimie de Lamarck ne concerne en fait qu'un aspect secondaire de sa pensée. Etre lavoisien ou non lavoisien affectait peu sa conception matérialiste de la vie. Après Lamarck, ceux qui ont utilisé la chimie lavoisienne pour expliquer la vie ne changeront en fait que les mots: employer d'une part les termes de "matière terreuse", ou d'autre part ceux d'"oxygène", d'"hydrogène", etc., ne sera en fait que faire appel à des "éléments" de base portant des noms certainement plus précis, mais en définitive seulement différents.

De plus, cette étude montre que Lamarck n'a pas eu à changer complètement sa conception matérialiste du monde en découvrant la possibilité de "produire" la vie à partir de la matière, mais seulement à compléter cette conception. Sans doute cette découverte a été importante, mais elle s'est opérée sans rupture fondamentale dans sa pensée. Elle a été seulement pour Lamarck l'occasion -ou la révélation- de la possibilité de boucler sa "physique terrestre".

Lamarck entend concentrer son attention davantage sur le développement de la vie que sur son origine. Le mouvement propre de la vie, qui est celui des fluides amène un perfectionnement graduel des êtres vivants. Lamarck explique ainsi ${ }^{52}$ que "parmi les animaux comme parmi les plantes, I'organisation (est) graduée dans ce qu'on peut appeler son perfectionnement". Nous sommes arrivés ainsi au bord de la découverte par Lamarck de la doctrine de l'evolution et de celle de la génération spontanée. "Si l'on considère la variété des moyens que la nature employe pour diversifier ses productions, c'est-à-dire les espèces et les sortes qui les constituent, on ne peut s'empêcher d'admirer les ressources infinies dont elle sçait faire usage pour arriver à son but. II semble en quelque sorte que tout ce qu'il est possible d'imaginer ait efíectivement lieu; que toutes les formes, toutes les facultés, et tous les modes

51 Ibid., p.254.

52 LAMARCK, J.B. (1797), p.314. 


\section{GOULVEN LAURENT}

aient été épuisées (sic) dans la formation et la composition de cette immense quantité de productions naturelles qui existent" 53 .

Ceci est sans doute une simple réminiscence de Buffon. Mais Lamarck va plus loin: "En effet, en considérant d'abord l'organisation animale la plus simple, pour s'élever ensuite graduellement jusqu'à celle qui est la plus composée, comme depuis la monade qui, pour ainsi dire, semble n'être qu'un point animé jusqu'aux animaux à mamelles, et parmi eux jusqu'à l'homme; il y a évidemment une gradation nuancée dans la composition de l'organisation et dans la nature de ses résultats, qu'on ne sçauroit trop admirer, et qu'on doit s'efforcer d'étudier, de déterminer et de bien connoître" (ibid.).

Comme on commence à l'entrevoir, c'est sans rupture que Lamarck va aboutir à sa nouvelle conception de la vie -une conception totalement matérialiste. C'est plutôt par un glissement naturel de sa pensée qu'il y parvient, un glissement tout à fait logique, etant donné l'option profondément matérialiste et mécaniciste de ses considérations sur la nature qu'il avait adoptée dans sa réflexion dès le point de départ. Du reste, les commentateurs ont toujours souligné la cohérence et l'unité de la pensée de Lamarck.

Diversité et complexification: les deux maîtres mots de la philosophie évolutionniste de Lamarck sont ainsi en place dès la fin des années 1790. Mais il reste encore à clore le système.

\section{La conception scientifique}

La vie devenait progressivement pour Lamarck un objet de sciencè, du moins dans son fonctionnement. II réalisait pour la vie ce que Newton avait fait pour le cosmos: une fois le mouvement donné au point de départ, le monde fonctionnait ensuite comme une machine réglée par les lois rigoureuses de la physique. Mais il fallait donner le mouvement, et c'est à ce point que l'on sortait du système mécaniciste, car il fallait avoir recours à un "primum movens". Chaque discipline scientifique en est d'ailleurs réduite à aboutir à une frontière, à sortir d'elle-même, ou du moins à mettre un point final à sa recherche des causes. II arrive en effet un moment où elle n'a plus de réponse dans sa propre sphère d'explication, et se heurte à un mur. La rencontre de cet obstacle est en général mal vécue par le savant. Ou il reconnaît ses limites, et en ce cas il laisse la voie ouverte à autre chose, c'est-à-dire à toutes les formes menaçantes de l'inconnu, y compris à un Etre suprême, qu'il peut nommer Dieu. Ou il ne le reconnaît pas, et alors il est obligé de forger des hypothèses, plus ou

53 «Discours préliminaire an 6-an 7» (1798-1799), dans Vachon, M., et al. (eds)(1972), J.-B. Lamarck Inédits, p.151. 
moins légitimes par définition, puisqu'il sort du domaine expérimental. Mais, de toutes manières, dans l'une ou l'autre option, il est impossible de fournir des preuves. Comme il n'y a pas de preuves de l'existence de l'inconnu, il n'y a pas non de preuves de sa non existence.

Dans le cheminement de Lamarck, nous sommes arrivés aux années 1800. Nous savons que c'est à cette époque qu'il "découvre", ou du moins qu'il construit scientifiquement, sa doctrine de l'évolution: les espèces vivantes se sont transformées au cours des siècles pour aboutir à la diversité biologique actuelle. Les forces physiques dont il dispose dans son système lui permettent d'expliquer ce développement. Le mouvement vital et les circonstances: voilà les facteurs de la montée et de la complication de l'arbre des vivants. A partir des formes les moins organisées et les plus "primitives", la vie s'est perfectionnée jusqu'à donner l'homme, le représentant le plus achevé des êtres vivants.

Mais il y a un butoir inférieur: I'apparition même de la vie. Jusque là, Lamarck s'était senti obligé de faire appel à un agent extérieur pour expliquer son apparition. II en était encore resté à une représentation fixiste de l'ordre du vivant. II était -il estimpossible en effet de se représenter l'apparition d'un être complexe, d'un éléphant par exemple, ou même seulement d'un insecte, en faisant seulement appel à de simples agents physiques. La science du xviiie siècle ne permettait pas d'expliquer l'apparition des vers dans la viande en faisant appel aux seules forces de la nature inanimée.

Mais dans une représentation du mondes actuel qui ne demande plus d'expliquer l'apparition des êtres par une création directe de chaque espèce, mais seulement par la lente montée de la vie à travers le cheminement progressif des êtres à travers des milliers de siècles, la question ne se pose plus de la même manière. La vie n'étant plus apparue dans un organisme complexe, mais dans un être à peine doué des caractères de la vie, dans une "matière à peine animalisée", il est beaucoup plus facile d'imaginer le passage direct de la matière à la vie.

Et c'est à ce point que Lamarck se rend compte qu'il dispose déjà dans son système des agents qui lui permettent d'expliquer le passage sans faire appel à un agent extérieur et surnaturel. Le concept de génération spontanée peut ainsi s'intégrer dans une explication matérialiste de l'univers.

\section{La génération spontanée}

A partir de 1797 déjà, nous avons rencontré chez Lamarck un concept qui a tendance à évacuer le concept métaphysique de la vie: c'est celui d'organisation qui deviendra chez lui tellement prépondérant qu'on le rencontrera plusieurs fois par page dans certains de ses ouvrages (dans l'index des Inédits, il est noté plus de 120 
fois). Lamarck annonce en effet ${ }^{54}$ qu'il va offrir "une classification générale des animaux, et une nouvelle distribution des animaux à sang blanc; celle précisément que j'enseigne dans mes cours. Ces distributions étant fondées sur l'organisation des animaux, il importait de les faire ici connaître". Du coup, pour ainsi dire, tout est ouvert pour une explication physique de la vie, car tout est déjà en place pour cela. Même si la définition de l'organisation reste encore, et restera, un peu floue -il s'agit pour Lamarck d'un "certain ordre de choses" - il lui suffira de réduire cette organisation à son degré le plus bas, au point zéro pour ainsi dire, pour passer de la vie à la matière, et, inversement, du non organique à l'organique, du non organisé à l'organisé, du non vivant au vivant, de la matière à la vie.

II en est ainsi dès 1800 , dès les premières affirmations de ce que l'on a appelé depuis la doctrine transformiste ou évolutionniste. Tout le début du projet présenté par Lamarck "sur l'origine des êtres vivans" mérite d'être cité ici, pour bien marquer l'enracinement et le développement de la théorie de la génération spontanée chez Lamarck ${ }^{55}$ : "Prouver d'abord le décroissement singulier qu'on observe dans la composition de l'organisation des animaux (et même des végétaux) en commençant par citer les animaux les plus parfaits c'est-à-dire ceux dont l'organisation est la plus composée et descendre successivement de classe en classe depuis les mammaux jusqu'aux polypes.

Prouver ensuite qu'à mesure que la composition de l'organisation diminue, les facultés animales sont graduellement moins nombreuses mais qu'elles acquièrent proportionnellement plus d'étendue.

Après cela établir que la nature dans sa marche n'a pas procédé du composé au simple, mais du simple au plus composé: ce qui est indiqué par la facilité avec laquelle elle fait les êtres organisés les plus simples et la lenteur et la difficulté avec lesquelles elle multiplie les êtres vivans les mieux ou les plus complettement organisés.

Ici définir la vie... etc.

Ensuite établir que la nature ayant formé immédiatement par la force des choses les corps vivans les plus simples, la diversité des circonstances et le temps ont dû faire varier les productions de la nature et donner lieu à toutes celles que nous observons.

Alors établir que ce n'est point la diversité des formes qui donne lieu aux habitudes, etc. mais que c'est au contraire la diversité des habitudes etc. qui occasionne celle des formes. Remonter alors depuis les animaux microscopiques jusqu'aux mammaux".

\footnotetext{
54 LAMARCK, J.B. (1797), p.314.

55 «Sur l'origine des êtres vivans» (1801-1803), dans VACHON, M., et al. (eds.)(1972), J. B. Lamarck Inédits, p. 180.
} 
A partir de cette idée claire de la montée progressive de la vie, si bien exposée dans ce "plan" de travail, Lamarck va entreprendre la mise en ordre de

son système d'explication de l'origine de cette vie. "On ne sçauroit douter maintenant que dans tout concours de circonstances favorables, des portions de matières inorganiques appropriées ne puissent par l'influence des 2 grands agens de la nature (la chaleur et l'humidité) recevoir dans leurs parties cette disposition qui ébauche l'organisation; de là conséquemment passer à l'état organique le plus simple, et dès lors jouir du 1 er élément de la vie".

Les deux grands agents de la nature: la chaleur et l'humidité, Lamarck les avait déjà utilisés pour expliquer le fonctionnement et la diversification de la vie. Maintenant il entrevoit que ces deux agents peuvent aussi servir à en expliquer l'origine.

Désormais la vie n'est plus pour Lamarck un concept métaphysique, même pas dans son apparition, comme elle l'avait été jusque là. Et Lamarck est conscient du progrès qu'il a fait dans sa propre conception de ce phénomène ${ }^{56}$ : "J'avoue que cela paroîtra inconcevable à tous ceux qui regardent la vie comme un principe quelconque, comme un être réel, en un mot comme quelque chose de particulier; tandis que dans la réalité la vie n'est qu'un mode ou qu'un ordre de choses relatives dans lequel tout changement qui détruit cet ordre, constitue ce qu'on nomme la mort". II n'y a plus besoin de sortir du domaine physique ${ }^{57}$ : "pour faire exister cette force vitale, dira Lamarck, et lui donner toutes les propriétés qu'on lui connoît, la nature n'a pas besoin de lois particulières; celles qui régissent généralement tous les corps lui suffisent parfaitement pour cet objet".

II y avait auparavant pour Lamarok une frontière entre le vivant et le non vivant. Désormais il n'y en a plus.

Ici encore Lamarck a soin de répondre aux objections qu'il s'était formulées à luimême, et que beaucoup allaient lui faire ${ }^{58}$ : "sans doute il n'est jamais arrivé, et il n'arrivera jamais que des matières non vivantes, quelles qu'elles soient, aient par un concours quelconque de circonstances, formé directement un lapin, un oiseau, un poisson, un insecte, et bien d'autres animaux dans lesquels l'organisation est déjà compliquée et fort avancée dans ses développemens. De pareils animaux n'ont assurément pu recevoir l'existence que par la voie de la génération et aucun fait d'animalisation ne peut les concerner".

Sa conception transformiste de l'histoire de la vie permet justement d'échapper définitivement à cette objection: "les premiers linéamens de l'organisation, les premières aptitudes à recevoir des développemens par intussusception, enfï les pre-

56 Ibid., p. 181

57 LAMARCK, J.B. (1809), ii, p.96.

58 «Sur l'origine des êtres vivans (1801-1803)», dans VACHON, M., et al. (eds.) (1972), J.-B. Lamarck Inédits, p. 181. 
mières ébauches de l'ordre et du mouvement qui constituent la vie, se forment tous les jours sous nos yeux, quoique jusqu'à présent nous n'y ayons jamais donné d'attention".

Pour résumer: "ces premiers linéamens de l'organisation, ces premières ébauches qui créent la vie, résultent à l'égard des matières appropriées qui les reçoivent, du concours de 2 causes principales: I'une active qui dispose tout et fournit le mouvement; et l'autre complettement passive, mais qui facilite tout et donne ce qui est nécessaire.

La première de ces causes réside dans l'action féconde de la chaleur, qu'avec raison l'on a appelée la mère des générations... La seconde des causes qui donnent lieu à la formation des premiers linéamens de l'organisation est l'humidité. Elle n'agit à la vérité que secondairement et subsidiairement. Mais elle donne et conserve la souplesse convenable aux parties qui par leur nature et la disposition que leur a donnée la première cause, sont destinées à contenir et à opérer des changemens sur les parties contenues. Ensuite elle entretient la masse de ces dernières et sert en même temps de véhicule aux particules propres par l'assimilation. à concourir au développement des parties contenantes et des organes".

Et pour bien situer cette explication dans le système mécaniste qui est le sien, Lamarck proclame comme une évidence ${ }^{59}$ : "Rien assurément de tout ce que je viens de dire ne répugne aux lois connues de la physique, ni aux connoissances que nous avons des facultés des matières dont il vient d'être question".

Une fois qu'il a acquis cette conviction, Lamarck ne variera plus dans l'exposition de sa théorie selon laquelle la vie a été produite directement par la nature. 11 ne fera ensuite que répéter, réaffirmer et réexposer sa vision matérialiste de l'apparition et du développement de la vie, dans ses Discours d'ouverture de ses cours (an vi (vii), an viii, an x, an xi, 1806, 1809, 1814, 1816), sa Philosophie zoologique (1809), son Introduction à l'Histoire Naturelle des Animaux sans Vertèbres (1815), et même jusque dans son Système analytique des connaissances positives de l'Homme (1820).

Le "calorique", qui faisait fonctionner le vivant, est aussi le principe de la $v^{60}{ }^{60}$ : "Cette matière subtile, pourvue de l'activité qu'elle est susceptible d'acquérir, devient le principe générateur de la vie, et en même temps celui qui l'entretient dans les corps qui en sont doués et qui les met dans le cas de la propager par la multiplication des individus.

La matière subtile et active dont il s'agit transforme en corps vivant du premier degré, soit animal soit végétal, les matières les plus souples à son action et les plus propres à recevoir dans de petites masses cet état de mouvement des parties dans

59 Ibid., p.182.

(6) Ibid., p.182. 
lequel la vie réside". Le feu (ou calorique, ou chaleur, ou électricité, comme l'on voudra), exerce donc ${ }^{61}$ une "influence pour ainsi dire créatrice".

La connaissance énorme et précise qu'il a acquise des Invertébrés (mot dont on sait qu'il fut l'inventeur) lui permet de donner un nom aux premiers êtres susceptibles d'avoir été "créés" de cette manière: sans doute "nous ne pouvons nous flatter de connoître les êtres étonnans qui constituent ce terme de la vie naissante, en un mot ce premier degré d'organisation qui suffit pour faire exister la vie, mais nous n'en sommes peut-être pas considérablement éloignés, lorsque parmi les animaux nous observons les monades, et parmi les végétaux certains mucors et même les Byssus pulvérulents".

Ce premier pas franchi de la création de la vie à partir de la matière, il n'y a plus de difficultés pour expliquer tout l'arbre des vivants dans sa diversité: "Les premières ébauches d'organisation une fois formées, la formation de tous les autres êtres doués de la vie n'est plus difficile à concevoir, quelle que soit la complication de leur organisation et le nombre de leurs facultés. Je l'ai déjà dit, du temps et une suffisante diversité de circonstances ont été dans le cas de produire tout ce qui existe à cet égard".

\section{Le fonctionnement de la vie}

II est clair, par ce que nous savons déjà des idées mécanicistes de Lamarck que, dans sa conception complétée de la nature de la vie, la fonction du vivant n'a pas à être fortement corrigée. En effet, le même principe fondamental du déterminisme continue à fonctionner ici, à plus forte raison maintenant que l'origine de la vie s'enracine directement dans la matière. "Tout fait ou phénomène observé dans un corps vivant, est à-la-fois un fait ou phénomène physique, et un produit de l'organisation"62. La vie, cette "puissance particulière", "agit toujours nécessairement, selon des lois régulatrices de tous ses actes... Elle agit toujours de la même manière, dans les mêmes circonstances"63. Si elle nous semble parfois agir au hasard, cette impression "n'exprime que notre ignorance des causes"64. En fait les moyens employés par la nature "forment ensemble une hiérarchie de puissances dans laquelle tout est lié, tout est dépendant, tout est en harmonie, tout est nécessaire"65. L'observateur est "forcé de reconnaître que la nature agit sans cesse, et toujours selon des lois qu'elle

\footnotetext{
61 Ibid., p.183.

62 LAMARCK, J.B. (1815-1822), I, Introduction, p. 12.

63 Ibid., p.310

64 Ibid., p.329

6.5 Ibid., p. 168.
} 


\section{GOULVEN LAURENT}

ne peut jamais transgresser"66. Devant ces faits, il ne peut donc "penser qu'il puisse y avoir quelque chose d'abstrait, quelque chose de métaphysique dans aucun de ses actes, dans une seule de ses opérations quelconques, et qu'elle ait quelque pouvoir sur des êtres non matériels". Tout ce que Lamarck avait déjà affirmé du fonctionnement de la vie, s'applique sans problème ni difficulté: les "fluides" calorique ou électrique, et les "circonstances", qui expliquaient toute la diversité du vivant, le font encore maintenant a fortiori. Mais le développement et la diversité ne se font pas sans transformations.

\section{Les transformations du vivant.}

Une des premières modifications subies par le vivant est, par le mouvement meme de la vie, de se complexifier. Le tableau de classification que Lamarck proposait dès 1797 était déjà assez éloquent à cet égard. A vrai dire, comme nous l'avons vu, tout est dit dans cette lecture de complexification croissante des êtres. Une fois cette idée acquise, elle va rester à la base de la vision transformiste de Lamarck. "En rangeant tous ceux (les animaux) que l'on a observés en une seule série d'après la considération de leurs rapports, et commençant la série par les plus imparfaits d'entre eux, on reconnoît que l'organisation est tellement simple dans les premiers, qu'ils ne possèdent pas même un seul organe spécial; mais qu'ensuite l'organisation se compose peu à peu et progressivement, de manière que les organes spéciaux sont formés l'un après l'autre, perfectionnés successivement, et qu'ils finissent par ëtre concentrés et cumulés dans les organisations les plus parfaites qui terminent l'autre extrémité de la série"67.

Là est l'ordre du tableau. Mais la nature a suivi elle-même cette marche ${ }^{68}$ "en procédant du plus simple vers le plus composé; car, ou assurément elle n'a rien opéré, ou, si les animaux font partie de ses productions, elle n'a point commencé par les plus composés et les plus parfaits". La "méthode naturelle", tout naturellement, "présentera une distribution dans laquelle on doit procéder du plus simple vers le plus composé"69.

La complexification des vivants n'est cependant pas linéaire, ni régulière. II peut y avoir dans cette marche des régressions et des avortements. II se produit en effet des "déviations... tant dans les classes que dans les familles de ces animaux, de la part du

66 Ibid.; p.219.

67 «Quelques considérations particulières nécessaires à l'avancement de la zoologie» (1808-1810), dans VACHON, M. et al. (eds.) (1972), J.-B. Lamarck Inédits, p.216.

68 LAMARCK, J.B. (1815-22), I, p.374.

69 Ibid., p. 375 . 


\section{LA BIOLOGIE DE LAMARCK}

produit des milieux habités, des climats, des moyens et des manières de vivre, en un mot des habitudes diverses de ces mêmes animaux, déviations qui s'opposent à la réalité d'une série simple..."70 L'ensemble des modifications se font dans les "organes non essentiels"; ils "tiennent à ce que ces organes sont plus soumis que les autres aux influences des circonstances extérieures, elles en entrainent de semblables dans la forme et dans l'état des parties les plus externes, et donne lieu à une diversité si considérable et si singulièrement ordonnée des espèces, qu'au lieu de les pouvoir ranger, comme les masses, en une série unique, simple et linéaire, sous la forme d'une échelle régulièrement graduée, ces mêmes espèces forment souvent autour des masses dont elles font partie, des ramifications latérales, dont les extrémités offrent des points véritablement isolés"71.

Le vivant est ainsi constamment soumis au changement, qui s'opère selon sa nature, et selon les circonstances. A chacune des deux causes qui provoquent les changements dans les êtres, Lamarck attribue une action spécifique. Le mouvement vital imprime un pertectionnement, par la complexification qu'il amène dans l'organisation. Les courants vitaux, nourriciers ou nerveux -La-marck parle de "fluides"- qui parcourent son corps ont pour effet de développer son organisation: "l'état d'organisation dans chaque corps vivant a été obtenu petit à petit par les progrès de l'influence du mouvement des fluides, et par ceux des changemens que ces fluides y ont continuellement subis dans leur nature et leur état par la succession habituelle de leurs déperditions et de leurs renouvellemens"72. Voilà pour le perfectionnement graduel, déjà cause de modifications. Les circonstances y ont contribué également. Elles ont un rôle particulier, rarement de perfectionnement, mais plus souvent soit de régression, soit de diversification. Si le milieu environnant est en effet hostile, I'organisation peut en être affectée et être amenée à régresser. Ainsi s'expliquent les avortements des ailes chez certains insectes, ou qu'ils restent à l'état de nymphes ou même de larves: "Ces particularités... sont dues à des avortemens de parties que la continuité des circonstances, qui tiennent à la manière de vivre de ces animaux, a perpétués et rendus habituels. Par des causes semblables, les cochenilles femelles sont aptères et sans élytres"73.

Mais les circonstances agissent plus souvent dans le sens d'une diversification des formes. "A l'aide de circonstances toujours diversifiées", la nature en effet sait opérer

\footnotetext{
70 «Discours d'ouverture pour le cours de 1816», dans VACHON, M. et al. (eds.) (1972), J.-B. Lamarck Inédits, p.131.

71 LAMARCK, J.B. (1906), p.506.

72 Ibid., p.487.

73 LAMARCK, J.B. (1815-1822), II, p.453-454.
} 


\section{GOULVEN LAURENT}

"des variations infinies" dans le monde des vivants ${ }^{74}$. Grâce à toutes ces causes et toutes ces influences, le monde actuel s'est mis en place: "du concours non interrompu de ces causes ou de ces loix de la nature, de beaucoup de temps et d'une diversité presqu'inconcevable de circonstances influentes, les corps vivans de tous les ordres ont été successivement formés"75.

\section{La production de l'Homme et de l'esprit}

Lamarck complète sa théorie de la vie par les deux extrémités: d'un côté il a iritroduit la génération spontanée pour relier la vie à la matière: la vie est un "produit" de la nature; de l'autre côté il soutient que l'intelligence est apparue à partir de la complexification de l'organisation physique de l'homme: l'"esprit" est aussi un "produit" de la nature.

Le fil conducteur de ce nouveau développement est toujours le mécanicisme matérialiste. Tout ce que "I'homme qui observe et réfléchit... aperçoit lui paraît pénétré de mouvement, soit effectif, soit contenu par des forces en équilibre. De tous côtés, il remarque, entre les corps, des actions réciproques et diverses, des réactions, des déplacemens, des agitations, des mutations de toutes les sortes, des altérations, des destructions, des formations nouvelles d'objets qui subissent à leur tour le sort d'autres semblables qui ont cessé d'exister, enfin, des reproductions constantes, mais assujéties aux influences des circonstances qui en font varier les résultats"76. Ainsi "tel est l'ensemble de choses qui constitue la nature, et dont nous sommes assurés de l'existence par l'observation; ... ensemble qui se compose de causes ou de forces toujours actives, toujours régularisées par des lois, et de moyens essentiels à la possibilité de leurs actions"77. Tout est en effet dans la nature, à laquelle il faut se soumettre et toujours revenir: "chaque fois que nous quitterons la nature pour nous livrer aux élans fantastiques de notre imagination, nous nous perdrons dans le vague, et les résultats de nos efforts ne seront que des erreurs. Les seules connoissances qu'il nous soit possible d'acquérir à son égard, sont et seront toujours uniquement celles que nous aurons puisées dans l'étude suivie de ses lois; hors de la nature, en un mot, tout n'est qu'égarement et mensonge: telle est mon opinion"78.

74 Lamarck, J.B. (1820), Système analytique des connaissances positives de l'homme, Belin, Paris, p.97.

75 LAMARCK, J.B. (1906), t.xl, p.487.

76 LAMARCK, J.B. (1815), i, p.326.

77 Ibid., p.327.

78 LAMARCK, J.B. (1809), ii, p.3. 
Tout est dans la nature, tout est par conséquent physique, jusqu'aux plus hautes facultés de l'homme. Lamarck pose en effet le principe général de la Biologie que "toutes les facultés, sans exception, sont complètement physiques, clest-à-dire, que chacune d'elles résulte essentiellement d'actes de l'organisation; en sorte qu'il sera facile de montrer comment de l'instinct le plus borné, dont la source peut etre aisément aperçue, la nature a pu parvenir à créer les facuités de l'intelligence, depuis celles qui sont les plus obscures, jusqu'à celles qui sont le plus développées"79.

L'homme rentre en effet dans la catégorie des etres produits par la nature: "Combien cette antiquité du globe terrestre s'agrandira encore aux yeux de l'homme, lorsqu'il se sera formé une juste idée de l'origine des corps vivans, ainsi que des causes du développement et du perfectionnement graduels de l'organisation de ces corps, et surtout lorsqu'il concevra que, le tems et les circonstances ayant été nécessaires pour dionner l'existence à toutes les espèces vivantes telles que nous les voyons actuellement, il est lui-même le dernier résultat et le maximum actuel de ce perfectionnement, dont le terme, stil en existe, ne peut être connu!"80. II a fallu du temps pour y parvenir: "Cet état particulier de l'organisation de l'homme a été acquis peu à peu à la suite de beaucoup de temps, à l'aide des circonstances qui s'y sont trouvées favorables"81.

La descendance de l'Homme à partir de l'animal, à partir d'un Singe, est nettement affirmée: "Effectivement, si une race quelconque de quadrumanes, surtout la plus perfectionnée d'entre elles, perdoit, par la nécessité des circonstances, ou par quelqu'autre cause, I'habitude de grimper sur les arbres, et d'en empoigner les branches avec les pieds, comme avec les mains, pour s'y accrocher; et si les individus de cette race, pendant une suite de générations, étoient forcés de ne se servir de leurs pieds que pour marcher, et cessoient d'employer leurs mains comme des pieds; il n'estpas douteux... que ces quadrumanes ne fussent à la fin transformes en bimanes"82 .

L'homme est rattaché par sa naissance au monde physique, et sa supériorité intellectuelle elle-même s'inscrit dans ce monde matériel: "La pensée, qui ne peut s'effectuer qu'à la suite d'idées préalablement acquises à l'aide des sens, et par l'impression du fluide nerveux qui les a successivement tracées au foyer de l'organe du sentiment, est un acte physique qui s'exécute dans ce foyer, c'est-à-dire dans le cerveau, qui le contient ou le compose" 83 .

L'homme rentre ainsi totalement dans la catégorie des animaux, dont les "facultés, quelles qu'elles soient, sont toutes des phénomènes organiques, purement physiques;

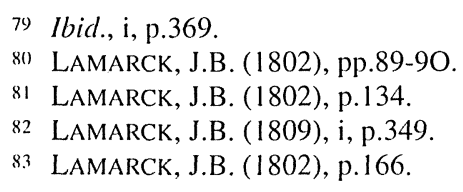




\section{GOULVEN LAURENT}

et ... aucune d'elles n'a rien de ce qu'on nomme métaphysique, qu'en vain l'on chercheroit dans la nature"84.

Lamarck revient ainsi toujours à la base de la connaissance: I'observation de la nature. L'homme ne peut en sortir: "Nous ne connaissons que des êtres physiques et que des objets relatifs à ces êtres: telle est la condition de notre nature. Si nos pensées, nos raisonnemens, nos principes, ont été considérés comme des objets métaphysiques, ces objets ne sont donc point des êtres"85. Aussi tous les phénomènes de la nature, et en particulier ceux relatifs à l'homme, et même son intelligence qui semble être d'une autre essence, sont des objets physiques: "ces phénomènes, qui lui paraissent si singuliers, si merveilleux, sont parfaitement organiques, toujours en rapport avec l'état de ses organes, nécessairement soumis au pouvoir ou aux lois de la nature"86.

L'esprit" n'existe donc pas aux yeux d'un naturaliste: c'est une faculté, mais ce n'est pas un être réel, c'est une création de l'imagination: "Je demanderai ce que c'est que cet être particulier qu'on nomme esprit...; être singulier qui est, dit-on, en rapport avec les actes du cerveau, de manière que les fonctions de cet organe sont d'un autre ordre que celles des autres organes de l'individu. Je ne vois dans cet être factice, dont la nature ne m'offre aucun modèle, qu'un moyen imaginé pour résoudre des difficultés que l'on n'avoit pu lever, faute d'avoir étudié suffisamment les lois de la nature"87. Et, pour bien montrer dans quelle catégorie il range ceux qui y croient, Lamarck ajoute -ce qui n'a pas dû faire plaisir à Cuvier, et qui montre qutil n'avait pas perdu son mordant avec l'age (il avait 76 ans à l'époque)-: "c'est à peu près la même chose que ces catastrophes universelles, auxquelles on a recours pour répondre à certaines questions géologiques qui nous embarrassent, parce que les procédés de la nature, dans les mutations de tous genres qu'elle produit sans cesse, ne sont point encore reconnus".

La doctrine de Lamarck - sa philosophie- est ainsi inscrite dans une discipline scientifique, la Biologie, qui n'a plus rien à emprunter à l'extérieur de la nature, de ses éléments matériels, ni de ses forces. A partir des éléments de base la terre, I'eau, I'air et le feu, qui lui fournissent les composants et le mouvement, Lamarck explique \{a vie et les vivants, depuis la matière brute sans vie jusqu'à la matière douée de la vie et meme de la forme la plus élevée de la vie qu'est l'intelligence. Ni la vie ni l'esprit ne sont plus désormais des objets "métaphysiques": sous ce nom ce sont des

\footnotetext{
84 "De l'intervalle considérable qui sépare la nature des corps inorganiques de celle des corps vivans; et parmi ceux-ci des caractères essentiels et exclusifs qui distinguent généralement les animaux des végétaux", dans Vachon, M. et al. (eds.)(1972), J.-B. Lamarck Inédits, p.286.

8.5 LAMARCK, J.B. (1820), p. 11.

86 Ibid, p.82.

87 LAMARCK, J.B. (1809), ii, p.172-173.
} 
objets irréels ou factices, créés par l'ignorance et maintenus par les préjugés des hommes.

\section{Conclusion}

La conception que Lamarck se fait de la vie, est, à partir de 1800, une conception unifiée et cohérent $^{88}$ qui mérite une appellation spécifiquel que Lamarck nomme Biologie. Pour lui, désormais, la vie est une propriété de la "matière", c'està-dire une "production de la nature". En effet, il n'a plus besoin d'un créateur particulier, ni d'un acte de création spéciale. II lui suffit d'avoir à sa disposition une matière terreuse humide, qu'il appeile "gélatineuse" ou "mucilagineuse", et une force qui la pénètre et la met en mouvement: le feu et l'électricité. On peut dire que cette construction intellectuelle est un mythe -et elle l'est bien évidemment. Mais le savant ne peut traiter de la vie comme une science s'il place à l'origine de la vie un acte spécial du Créateur. A moins de jouer à cache-cache avec les mots et la réalité -comme le fera Darwin. Deux cents ans après Lamarck, on n'a pas encore dépassé ce stade du mythe de la production directe de la vie à partir de la matière. II est clair en effet qu'il faut aller jusqu'à reconnaître cette infirmité intellectuelle de la pensée.

Dès 1803, Lamarck est à même de proposer une doctrine cohérente d'ensemble ${ }^{89}$ "la nature, écrit-il, opère elle-mëme tous les prodiges qu'on vient de citer; ... elle crée l'organisation, la vie, le sentiment même; ... elle multiplie et diversifie, dans des limites qui ne nous sont pas connues, les organes et les facultés des corps organisés dont elle soutient ou propage l'existence; ... elle crée dans les animaux, par la seule voie du besoin qui établit et dirige les habitudes, la source de toutes les actions, depuis les plus simples jusqu'à celles qui constituent l'instinct, I'industrie, enfin le raisonnementt". Ainsi "la nature possède, dans ses facultés, tout ce qui est nécessaire pour avoir pu produire elle-même ce que nous admirons en elle", et c'est ce que Lamarck "compte prouver dans (sa) Biologie"90. Même s'il n'a pas eu le temps d'écrire cet ouvrage, il n'en reste pas moins qu'il en a exposé tous les éléments. Les "vérités claires, évidentes, sur lesquelles les observations ne peuvent autoriser le moindre doute, lorsqu'on voudra les examiner" consistent, selon Lamarck ${ }^{91}$, dans les "principes fondamentaux suivans:

\footnotetext{
88 Voir la récente et remarquable étude de BARSANTI, G. (1995), «La naissance de la biologie», dans Nature, Histoire, Société, Essais en hommage à Jacques Roger, Paris, Klincksieck, p. 197-228.

89 LAMARCK, J.B. (1906), p.538.

9) Ibid., p.539.

91 "Discours d'ouverture du cours de 1814", dans VACHON, M. et al. (eds.)(1972), J.-B. Lamarck Inédits, pp.232-233.
} 


\section{GOULVEN LAURENT}

... ler principe: Tout fait ou phénomène que l'observation peut faire connoître, est essentiellement physique, et ne doit son existence ou sa production qu'à des corps, ou qu'à des relations entre des corps.

$2 d$ principe: tout mouvement ou changement, toute force agissante, et tout effet quelconque, observés dans un corps tiennent nécessairement à des causes mécaniques, régies par des lois.

3e principe: tout fait ou phénomène observé dans un corps vivant, est à la fois un fait ou phénomène physique, et un produit de l'organisation.

4e principe: il n'y a dans la nature aucune matière qui ait en propre la faculté de vivre. Tout corps en qui la vie se manifeste, offre dans le produit de l'organisation qu'il possède, et dans celui d'une suite de mouvemens excités dans ses parties, le phénomène physique et organique que la vie constitue, phénomène qui s'exécute et se maintient dans ce corps, tant que les conditions essentielles à sa production subsistent.

5e principe: il n'y a dans la nature aucune matière qui ait en propre la faculté d'avoir ou de se former des idées, d'exécuter des opérations entre des idées, en un mot, de penser. Là où de pareils phénomènes se montrent, (et l'on en observe de cette sorte dans les animaux les plus parfaits) I'on trouve toujours un système d'organes particulier propre à les produire; système dont l'étendue et l'intégrité sont constamment en rapport avec le degré d'éminence et l'état des phénomènes dont il s'agit.

6e principe: enfin, il n'y a dans la nature aucune matière qui ait en propre la faculté de sentir. Aussi, là où cette faculté peut etre constatée, là seulement se trouve, dans le corps vivant qui en est doué, un système d'organes particulier capable de donner lieu au phénomène physique, mécanique et organique qui, seul, constitue la sensation".

Enfin, Lamarck éprouve le besoin d'ajouter: "A ces principes, à l'abri de toute contestation solide, et sans lesquels la zoologie seroit sans fondemens, j'ajouterai:

$1^{\circ}$ qu'il y a toujours un rapport parfait entre l'état, soit d'intégrité ou d'altération, soit d'étendue ou de perfectionnement d'une faculté organique, et celui de l'organe ou du système d'organes qui la produit;

$2^{\circ}$ que, plus une faculté organique est éminente, plus l'organisation à laquelle appartient le système d'organes qui y donne lieu, est composée".

La Biologie de Lamarck n'est certes pas la première doctrine matérialiste; mais c'est la première qui ait su synthétiser les données évolutionnistes et les théories matérialistes dans un système cohérent. C'est la première doctrine évolutionniste matérialiste. C'est peut-être d'ailleurs la seule digne de ce nom qui ait été élaborée, jusqu'à la doctrine synthétique de l'Evolution, des années 1930. 